\title{
On Some Inequalities of Uncertainty Principles Type in Quantum Calculus
}

\author{
Ahmed Fitouhi, ${ }^{1}$ Néji Bettaibi, ${ }^{2}$ Rym H. Bettaieb, ${ }^{2}$ and Wafa Binous ${ }^{3}$ \\ ${ }^{1}$ Faculté des Sciences de Tunis, Université de Tunis El Manar, 1060 Tunis, Tunisia \\ ${ }^{2}$ Institut Préparatoire aux Études d'Ingénieur de Monastir, Université de Monastir, \\ 5000 Monastir, Tunisia \\ ${ }^{3}$ Institut de Biotechnologie, Université de Jendouba, 9000 Béja, Tunisia
}

Correspondence should be addressed to Néji Bettaibi, neji.bettaibi@ipein.rnu.tn

Received 19 July 2007; Revised 31 January 2008; Accepted 14 April 2008

Recommended by Wolfgang Castell

The aim of this paper is to generalize the $q$-Heisenberg uncertainty principles studied by Bettaibi et al. (2007), to state local uncertainty principles for the $q$-Fourier-cosine, the $q$-Fourier-sine, and the $q$-Bessel-Fourier transforms, then to provide an inequality of Heisenberg-Weyl-type for the $q$ Bessel-Fourier transform.

Copyright (C) 2008 Ahmed Fitouhi et al. This is an open access article distributed under the Creative Commons Attribution License, which permits unrestricted use, distribution, and reproduction in any medium, provided the original work is properly cited.

\section{Introduction}

The uncertainty principle is a metatheorem in harmonic analysis that asserts, with the use of some inequalities, that a function and its Fourier transform cannot be sharply localized. We refer to the survey article by Folland and Sitaram [1] and the book of Havin and Jöricke [2] for various classical uncertainty principles of different nature which may be found in the literature.

In [3], the authors gave $q$-analogues of the Heisenberg uncertainty principle for the $q$ Fourier-cosine and the $q$-Fourier-sine transforms. One of the aims of this paper is to provide a generalization of their work next to state local uncertainty principles for various $q$-Fourier transforms.

This paper is organized as follows. In Section 2, we present some preliminaries results and notations that will be useful in the sequel. In Section 3, we prove a density theorem and a $q$-analogue of the Hausdorff-Young inequality. Then, we state a generalization of the $q$ Heisenberg uncertainty principle for the $q$-Fourier-cosine and the $q$-Fourier-sine transforms. In Section 4, we state local uncertainty principles for the $q$-Fourier-cosine, $q$-Fourier-sine, and 
$q$-Bessel-Fourier transforms. Then, we give a Heisenberg-Weyl-type inequality for some $q$ Bessel-Fourier transform.

\section{Notations and preliminaries}

Throughout this paper, we assume $q \in] 0,1[$. We recall some usual notions and notations used in the $q$-theory (see $[4,5]$ ). We refer to the book by Gasper and Rahman [4] for the definitions, notations, and properties of the $q$-shifted factorials and the $q$-hypergeometric functions.

We write $\mathbb{R}_{q}=\left\{ \pm q^{n}: n \in \mathbb{Z}\right\}, \mathbb{R}_{q,+}=\left\{q^{n}: n \in \mathbb{Z}\right\}$, and

$$
[x]_{q}=\frac{1-q^{x}}{1-q}, \quad x \in \mathbb{C}, \quad[n]_{q} !=\frac{(q ; q)_{n}}{(1-q)^{n}}, \quad n \in \mathbb{N} .
$$

The $q$-derivative of a function $f$ is given by

$$
\left(D_{q} f\right)(x)=\frac{f(x)-f(q x)}{(1-q) x} \quad \text { if } x \neq 0,
$$

$\left(D_{q} f\right)(0)=\lim _{k \rightarrow+\infty}\left(D_{q} f\right)\left(q^{k}\right)$, provided that the limit exists.

The $q$-Jackson integrals from 0 to $a$ and from 0 to $\infty$, of a function $f$, are (see [6])

$$
\int_{0}^{a} f(x) d_{q} x=(1-q) a \sum_{n=0}^{\infty} f\left(a q^{n}\right) q^{n}, \quad \int_{0}^{\infty} f(x) d_{q} x=(1-q) \sum_{n=-\infty}^{\infty} f\left(q^{n}\right) q^{n},
$$

provided that the sums converge absolutely.

The $q$-Jackson integral in a generic interval $[a, b]$ is given by (see [6])

$$
\int_{a}^{b} f(x) d_{q} x=\int_{0}^{b} f(x) d_{q} x-\int_{0}^{a} f(x) d_{q} x .
$$

The $q$-integration by parts rule is given, for suitable functions $f$ and $g$, by

$$
\int_{a}^{b} g(x) D_{q} f(x) d_{q} x=f(b) g(b)-f(a) g(a)-\int_{a}^{b} f(q x) D_{q} g(x) d_{q} x .
$$

Jackson (see [6]) defined a $q$-analogue of the Gamma function by

$$
\Gamma_{q}(x)=\frac{(q ; q)_{\infty}}{\left(q^{x} ; q\right)_{\infty}}(1-q)^{1-x}, \quad x \neq 0,-1,-2, \ldots
$$

The third Jackson $q$-Bessel function (see $[7,8]$ ) is

$$
J_{v}\left(z ; q^{2}\right)=\frac{z^{v}}{\left(1-q^{2}\right)^{v} \Gamma_{q^{2}}(v+1)}{ }_{1} \varphi_{1}\left(0 ; q^{2 v+2} ; q^{2}, q^{2} z^{2}\right),
$$


and the $q$-trigonometric functions ( $q$-cosine and $q$-sine) are defined by (see [9])

$$
\begin{aligned}
& \cos \left(x ; q^{2}\right)=\frac{\Gamma_{q^{2}}(1 / 2)}{q\left(1+q^{-1}\right)^{1 / 2}} x^{1 / 2} J_{-1 / 2}\left(\frac{1-q}{q} x ; q^{2}\right)=\sum_{n=0}^{\infty}(-1)^{n} q^{n(n-1)} \frac{x^{2 n}}{[2 n]_{q} !}, \\
& \sin \left(x ; q^{2}\right)=\frac{\Gamma_{q^{2}}(1 / 2)}{\left(1+q^{-1}\right)^{1 / 2}} x^{1 / 2} J_{1 / 2}\left(\frac{1-q}{q} x ; q^{2}\right)=\sum_{n=0}^{\infty}(-1)^{n} q^{n(n-1)} \frac{x^{2 n+1}}{[2 n+1]_{q} !} .
\end{aligned}
$$

They verify

$$
D_{q} \cos \left(x ; q^{2}\right)=-\frac{1}{q} \sin \left(q x ; q^{2}\right), \quad D_{q} \sin \left(x ; q^{2}\right)=\cos \left(x ; q^{2}\right)
$$

We need the following spaces and norms.

(i) $\mathcal{S}_{* q}\left(\mathbb{R}_{q}\right)$ is the space of even functions $f$ on $\mathbb{R}_{q}$ satisfying

$$
\forall n, m \in \mathbb{N}, \quad P_{n, m, q}(f)=\sup _{x \in \mathbb{R}_{q} ; 0 \leq k \leq n}\left|\left(1+x^{2}\right)^{m} D_{q}^{k} f(x)\right|<+\infty
$$

(ii) $L_{q}^{n}\left(\mathbb{R}_{q,+}, x^{2 v+1} d_{q} x\right), n \geq 1, v \geq-1 / 2$, is the set of all functions defined on $\mathbb{R}_{q,+}$ such that

$$
\|f\|_{n, v, q}=\left\{\int_{0}^{\infty}|f(x)|^{n} x^{2 v+1} d_{q} x\right\}^{1 / n}<\infty
$$

(iii) $L_{q}^{n}\left(\mathbb{R}_{q,+}\right)=L_{q}^{n}\left(\mathbb{R}_{q,+}, d_{q} x\right), n \geq 1$, and $\|\cdot\|_{n, q}=\|\cdot\|_{n,-1 / 2, q}$.

(iv) $L_{q}^{\infty}\left(\mathbb{R}_{q,+}\right)$ is the set of all bounded functions on $\mathbb{R}_{q,+}$. We write $\|f\|_{\infty, q}=\sup _{x \in \mathbb{R}_{q,+}}|f(x)|$.

\section{Generalization of the Heisenberg uncertainty principle}

The $q$-Fourier-cosine and the $q$-Fourier-sine transforms are defined as (see $[8,9]$ )

$$
\mathcal{F}_{q}(f)(x)=c_{q} \int_{0}^{\infty} f(t) \cos \left(x t ; q^{2}\right) d_{q} t, \quad{ }_{q} \mathcal{F}(f)(x)=c_{q} \int_{0}^{\infty} f(t) \sin \left(x t ; q^{2}\right) d_{q} t,
$$

where

$$
c_{q}=\frac{\left(1+q^{-1}\right)^{1 / 2}}{\Gamma_{q^{2}}(1 / 2)} .
$$

Letting $q \uparrow 1$ subject to the condition $(\log (1-q) / \log (q)) \in \mathbb{Z}$ gives, at least formally, the classical Fourier transforms (see $[3,10])$. In the remainder of the present paper, we assume that this condition holds. 
It was shown in $[8,9]$ that we have the following result.

Proposition 3.1. (1) For $f \in L_{q}^{1}\left(\mathbb{R}_{q,+}\right)$, one has $\mathcal{F}_{q}(f) \in L_{q}^{\infty}\left(\mathbb{R}_{q,+}\right)$ and

$$
\left\|\mathcal{F}_{q}(f)\right\|_{\infty, q} \leq \frac{\left(1+q^{-1}\right)^{1 / 2}}{\Gamma_{q^{2}}(1 / 2)(q ; q)_{\infty}^{2}}\|f\|_{1, q} .
$$

(2) $\mathcal{F}_{q}$ is an isomorphism of $L_{q}^{2}\left(\mathbb{R}_{q,+}\right)$ (resp., $S_{*, q}\left(\mathbb{R}_{q}\right)$ ) onto itself. Moreover, one has $\mathcal{F}_{q}^{-1}=\mathcal{F}_{q}$ and the following Plancherel formula:

$$
\left\|\mathcal{F}_{q}(f)\right\|_{2, q}=\|f\|_{2, q}, \quad f \in L_{q}^{2}\left(\mathbb{R}_{q,+}\right)
$$

Similarly, it was shown in $[3,8]$ that the $q$-Fourier-sine transform verifies the following properties.

Proposition 3.2. (1) For $f \in L_{q}^{1}\left(\mathbb{R}_{q,+}\right)$, one has ${ }_{q} \mathcal{F}(f) \in L_{q}^{\infty}\left(\mathbb{R}_{q,+}\right)$ and

$$
\left\|_{q} \mathcal{F}(f)\right\|_{\infty, q} \leq \frac{\left(1+q^{-1}\right)^{1 / 2}}{\Gamma_{q^{2}}(1 / 2)(q ; q)_{\infty}^{2}}\|f\|_{1, q} .
$$

(2) ${ }_{q} \mathcal{F}$ is an isomorphism of $L_{q}^{2}\left(\mathbb{R}_{q,+}\right)$ onto itself; its inverse is given by ${ }_{q} \mathcal{F}^{-1}=\left(1 / q^{2}\right)_{q} \mathcal{F}$. One has the following Plancherel formula:

$$
\left\|_{q} \mathcal{F}(f)\right\|_{2, q}=q\|f\|_{2, q}, \quad f \in L_{q}^{2}\left(\mathbb{R}_{q,+}\right) .
$$

Let us now state the following useful density result.

Proposition 3.3. For all $n \geq 1, S_{*, q}\left(\mathbb{R}_{q}\right)$ is dense in $L_{q}^{n}\left(\mathbb{R}_{q,+}\right)$.

Proof. Let $n \geq 1$ and $f \in L_{q}^{n}\left(\mathbb{R}_{q,+}\right)$. For $p \in \mathbb{N}$, put $f_{p}=f \cdot X\left[q^{p}, q^{-p}\right]$, where $\chi_{\left[q^{p}, q^{-p}\right]}$ is the characteristic function of $\left[q^{p}, q^{-p}\right]$.

It is clear that for all $p \in \mathbb{N}, f_{p} \in S_{*, q}\left(\mathbb{R}_{q}\right)$ and $\left|f-f_{p}\right|^{n} \leq|f|^{n}$. So, the Lebesgue theorem implies that $\left(f_{p}\right)_{p}$ converges to $f$ in $L_{q}^{n}\left(\mathbb{R}_{q,+}\right)$.

Remark 3.4. Using the density of $S_{*, q}\left(\mathbb{R}_{q}\right)$ in $L_{q}^{n}\left(\mathbb{R}_{q,+}\right)(n \geq 1)$, one can see that the $q$-Fouriercosine (resp., $q$-Fourier-sine) transform has a unique continuous extension on $L_{q}^{n}\left(\mathbb{R}_{q,+}\right)$, that will also be denoted as $\mathcal{F}_{q}$ (resp., $q \mathcal{F}$ ). We have the following $q$-analogue of the HausdorffYoung inequality.

Theorem 3.5. Let $n \in] 1,2]$ (resp., $n=1)$ and $m=n /(n-1)$ (resp., $m=\infty$ ) be the dual exponent of $n$. For all $f$ in $L_{q}^{n}\left(\mathbb{R}_{q,+}\right)$, the functions $\mathcal{F}_{q}(f)$ and ${ }_{q} \mathcal{F}(f)$ belong to $L_{q}^{m}\left(\mathbb{R}_{q,+}\right)$, and one has

$$
\left\|\mathcal{F}_{q}(f)\right\|_{m, q} \leq C_{1}\|f\|_{n, q}, \quad\left\|_{q} \mathcal{F}(f)\right\|_{m, q} \leq C_{2}\|f\|_{n, q},
$$


where

$$
C_{1}=\left(\frac{\left(1+q^{-1}\right)^{1 / 2}}{\Gamma_{q^{2}}(1 / 2)(q ; q)_{\infty}^{2}}\right)^{1-2((n-1) / n)}, \quad C_{2}=\left(\frac{\left(1+q^{-1}\right)^{1 / 2}}{\Gamma_{q^{2}}(1 / 2)(q ; q)_{\infty}^{2}}\right)^{1-2((n-1) / n)} q^{2((n-1) / n)} .
$$

Proof. The result is a direct consequence of [11, Theorem 1.3.4, page 35], and Propositions 3.1 and 3.2, by taking $S_{*, q}\left(\mathbb{R}_{q}\right)$ as a set of simple functions. forms.

The following lemma gives relations between the two Fourier $q$-trigonometric trans-

Lemma 3.6. (1) For $f \in L_{q}^{2}\left(\mathbb{R}_{q,+}\right)$ such that $D_{q} f \in L_{q}^{2}\left(\mathbb{R}_{q,+}\right)$, one has

$$
{ }_{q} \mathcal{F}\left(D_{q} f\right)(\lambda)=-\frac{\lambda}{q} \mathcal{F}_{q}(f)\left(\frac{\lambda}{q}\right), \quad \lambda \in \mathbb{R}_{q,+} .
$$

(2) Additionally, if $\lim _{n \rightarrow+\infty} f\left(q^{n}\right)=0$, then

$$
\mathcal{F}_{q}\left(D_{q} f\right)(\lambda)=\frac{\lambda}{q^{2}} q \mathcal{F}(f)(\lambda), \quad \lambda \in \mathbb{R}_{q,+} .
$$

Proof. The same steps as in the proof of [3, Lemma 2]; the $q$-integration by parts rule and the fact that

$$
\int_{0}^{\infty} f(t) d_{q} t=\lim _{n \rightarrow+\infty} \int_{q^{n}}^{q^{-n}} f(t) d_{q} t
$$

give the result. principle.

In [3], the authors proved the following $q$-analogues of the Heisenberg uncertainty

Theorem 3.7. Let $f$ be in $L_{q}^{2}\left(\mathbb{R}_{q,+}\right)$ such that $D_{q} f$ is in $L_{q}^{2}\left(\mathbb{R}_{q,+}\right)$. Then,

$$
\|t f\|_{2, q}\left\|\lambda \mathcal{F}_{q}(f)\right\|_{2, q} \geq \frac{q}{q^{3 / 2}+1}\|f\|_{2, q}^{2} .
$$

In addition, if $\lim _{n \rightarrow+\infty} f\left(q^{n}\right)=0$, one has

$$
\|t f\|_{2, q}\left\|\lambda_{q} \mathcal{F}(f)\right\|_{2, q} \geq \frac{q}{q^{-3 / 2}+1}\|f\|_{2, q}^{2} .
$$

Now, we are in a position to generalize Theorem 3.7. One obvious way to generalize it is to replace the $L_{q}^{2}$ norms by $L_{q}^{n}$ norms. This is the purpose of the following result.

Theorem 3.8. For $1 \leq n \leq 2$ and $f \in L_{q}^{2}\left(\mathbb{R}_{q,+}\right)$, one has

$$
\begin{aligned}
& \|f\|_{2, q}^{2} \leq C_{1}^{\prime}\|x f\|_{n, q}\left\|\lambda \mathcal{F}_{q}(f)\right\|_{n, q}, \\
& \|f\|_{2, q}^{2} \leq C_{2}^{\prime}\|x f\|_{n, q}\left\|\lambda_{q} \mathcal{F}(f)\right\|_{n, q},
\end{aligned}
$$


where

$$
C_{1}^{\prime}=q^{-1+1 / n}\left(1+q^{-(n+1) / n}\right) C_{2}, \quad C_{2}^{\prime}=q^{-1}\left(1+q^{-(n+1) / n}\right) C_{1},
$$

with $C_{1}$ and $C_{2}$ being given by (3.8).

Proof. The case $n=2$ has been dealt with in Theorem 3.7. Now, assume $1 \leq n<2$ and let $m$ be the dual exponent of $n$. Let $f \in S_{*, q}\left(\mathbb{R}_{q}\right)$ such that $\lim _{t \rightarrow 0} f(t)=0$. From the relation

$$
D_{q}(f \bar{f})(t)=D_{q} f(t) \bar{f}(t)+f(q t) D_{q} \bar{f}(t),
$$

the $q$-integration by parts rule, and the Hölder inequality, we have, since $t|f(t)|^{2}$ tends to 0 as $t$ tends to $\infty$ in $\mathbb{R}_{q,+}$,

$$
\begin{aligned}
\frac{1}{q} \int_{0}^{\infty}|f(t)|^{2} d_{q} t= & \left|\int_{0}^{\infty} t D_{q}(f \bar{f})(t) d_{q} t\right| \\
\leq & \int_{0}^{\infty}\left|t D_{q} f(t) \bar{f}(t)\right| d_{q} t+\int_{0}^{\infty}\left|t f(q t) D_{q} \bar{f}(t)\right| d_{q} t \\
\leq & \left(\int_{0}^{\infty}|t \bar{f}(t)|^{n} d_{q} t\right)^{1 / n}\left(\int_{0}^{\infty}\left|D_{q} f(t)\right|^{m} d_{q} t\right)^{1 / m} \\
& +\left(\int_{0}^{\infty}|t f(q t)|^{n} d_{q} t\right)^{1 / n}\left(\int_{0}^{\infty}\left|D_{q} \bar{f}(t)\right|^{m} d_{q} t\right)^{1 / m} .
\end{aligned}
$$

However, the change of variable $u=q t$ gives

$$
\left(\int_{0}^{\infty}|t f(q t)|^{n} d_{q} t\right)^{1 / n}=q^{-(n+1) / n}\left(\int_{0}^{\infty}|t f(t)|^{n} d_{q} t\right)^{1 / n} .
$$

So,

$$
\frac{1}{q} \int_{0}^{\infty}|f(t)|^{2} d_{q} t \leq\left(1+q^{-(n+1) / n}\right)\|t f\|_{n, q}\left\|D_{q}(f)\right\|_{m, q} .
$$

On the other hand, we have $D_{q}(f)=\mathcal{F}_{q}\left[\mathcal{F}_{q}\left(D_{q}(f)\right)\right]=q^{-2}{ }_{q} \mathcal{F}\left[q \mathcal{F}\left(D_{q}(f)\right)\right]$ since $D_{q}(f)$ is in $L_{q}^{2}\left(\mathbb{R}_{q,+}\right)$. Then, by using Lemma 3.6 and the $q$-analogue of the Hausdorff-Young inequality, we obtain

$$
\begin{aligned}
& \left\|D_{q}(f)\right\|_{m, q} \leq C_{1}\left\|\mathcal{F}_{q}\left(D_{q}(f)\right)\right\|_{n, q}=\frac{C_{1}}{q^{2}}\left\|\lambda_{q} \mathcal{F}(f)\right\|_{n, q^{\prime}} \\
& \left\|D_{q}(f)\right\|_{m, q} \leq q^{-2} C_{2}\left\|_{q} \mathcal{F}\left(D_{q}(f)\right)\right\|_{n, q}=q^{-2} C_{2}\left\|\frac{\lambda}{q} \mathcal{F}_{q}(f)\left(\frac{\lambda}{q}\right)\right\|_{n, q}=q^{-2+1 / n} C_{2}\left\|\lambda \mathcal{F}_{q}(f)\right\|_{n, q^{\circ}} .
\end{aligned}
$$

Thus,

$$
\begin{aligned}
& \|f\|_{2, q}^{2} \leq q^{-1}\left(1+q^{-(n+1) / n}\right) C_{1}\|t f\|_{n, q}\left\|\lambda_{q} \mathcal{F}(f)\right\|_{n, q^{\prime}} \\
& \|f\|_{2, q}^{2} \leq q^{-1+1 / n}\left(1+q^{-(n+1) / n}\right) C_{2}\|t f\|_{n, q}\left\|\lambda \mathcal{F}_{q}(f)\right\|_{n, q^{\circ}} .
\end{aligned}
$$

Now, let $f \in L_{q}^{2}\left(\mathbb{R}_{q,+}\right)$; it is easy to see that for all $p \in \mathbb{N}, f_{p}=f \chi_{\left[q^{p}, q^{-p}\right]} \in S_{*, q}\left(\mathbb{R}_{q}\right), \lim _{t \rightarrow 0} f_{p}(t)=0$, and $\left(f_{p}\right)_{p}$ converges to $f$ in $L_{q}^{2}\left(\mathbb{R}_{q,+}\right)$. Moreover, if the right-hand side of (3.14) (resp., (3.15)) is finite, then the functions $t f$ and $\lambda \mathcal{F}_{q}(f)$ (resp., $\lambda_{q} \mathcal{F}(f)$ ) are in $L_{q}^{n}\left(\mathbb{R}_{q,+}\right)$, and they are limits in $L_{q}^{n}\left(\mathbb{R}_{q,+}\right.$ ) (as $p$ tends to $\infty$ ) of $t f_{p}$ and $\lambda \mathcal{F}_{q}\left(f_{p}\right)$ (resp., $\lambda_{q} \mathcal{F}\left(f_{p}\right)$ ), respectively. Finally, the substitution of $f_{p}$ in (3.22) and a passage to the limit when $p$ tends to $\infty$ complete the proof. 


\section{Local uncertainty principles}

In the literature, the first classical local inequalities were obtained by Faris (see [12]) in 1978, and they were generalized by Price (see $[13,14])$ in 1983 and 1987. In this section, we will generalize Price's results by giving their $q$-analogues.

\subsection{Local uncertainty principles for the q-Fourier trigonometric transforms}

Theorem 4.1. If $0<a<1 / 2$, there is a constant $K=K(a, q)$ such that for all bounded subset $E$ of $\mathbb{R}_{q,+}$ and all $f \in L_{q}^{2}\left(\mathbb{R}_{q,+}\right)$, one has

$$
\int_{E}\left|\mathcal{F}_{q}(f)(\lambda)\right|^{2} d_{q} \lambda \leq K|E|^{2 a}\left\|x^{a} f\right\|_{2, q}^{2}
$$

Here, $|E|=\int_{0}^{\infty} X_{E}(x) d_{q} x$ and $K=\left(\left(\tilde{c}_{q} / \sqrt{[1-2 a]_{q}}\right)((1-2 a) / 2 a)\right)^{4 a}\left(1 /(1-2 a)^{2}\right)$, where $\tilde{c}_{q}=$ $\left(1+q^{-1}\right)^{1 / 2} / \Gamma_{q^{2}}(1 / 2)(q ; q)_{\infty}^{2}$.

Proof. For $r>0$, let $\chi_{r}=X_{[0, r]}$ be the characteristic function of $[0, r]$ and $\tilde{X}_{r}=1-X_{r}$.

Then, for $r>0$, we have, since $f \cdot x_{r} \in L_{q}^{1}\left(\mathbb{R}_{q,+}\right)$,

$$
\begin{aligned}
\left(\int_{E}\left|\mathcal{F}_{q}(f)(\lambda)\right|^{2} d_{q} \lambda\right)^{1 / 2} & =\left\|\mathscr{F}_{q}(f) \chi_{E}\right\|_{2, q} \leq\left\|\mathscr{F}_{q}\left(f \cdot X_{r}\right) X_{E}\right\|_{2, q}+\left\|\mathscr{F}_{q}\left(f \cdot \tilde{x}_{r}\right) \chi_{E}\right\|_{2, q} \\
& \leq|E|^{1 / 2}\left\|\mathscr{F}_{q}\left(f \cdot x_{r}\right)\right\|_{\infty, q}+\left\|\mathcal{F}_{q}\left(f \cdot \tilde{X}_{r}\right)\right\|_{2, q^{\prime}}
\end{aligned}
$$

and by the use of the Hölder inequality, we obtain

$$
\begin{aligned}
& \left\|\mathcal{F}_{q}\left(f \cdot x_{r}\right)\right\|_{\infty, q} \leq \tilde{c}_{q}\left\|f \cdot X_{r}\right\|_{1, q} \\
& \quad=\tilde{c}_{q}\left\|x^{-a} X_{r} \cdot x^{a} f\right\|_{1, q} \leq \tilde{c}_{q}\left\|x^{-a} X_{r}\right\|_{2, q}\left\|x^{a} f\right\|_{2, q} \leq \frac{\tilde{c}_{q}}{\sqrt{[1-2 a]_{q}}} r^{1 / 2-a}\left\|x^{a} f\right\|_{2, q} .
\end{aligned}
$$

On the other hand, since $f \in L_{q}^{2}\left(\mathbb{R}_{q,+}\right)$, we have $f \cdot \tilde{x}_{r} \in L_{q}^{2}\left(\mathbb{R}_{q,+}\right)$, and by the Plancherel formula, we get

$$
\left\|\mathcal{F}_{q}\left(f \cdot \tilde{X}_{r}\right)\right\|_{2, q}=\left\|f \cdot \tilde{X}_{r}\right\|_{2, q}=\left\|x^{-a} \tilde{X}_{r} \cdot x^{a} f\right\|_{2, q} \leq\left\|x^{-a} \tilde{X}_{r}\right\|_{\infty, q}\left\|x^{a} f\right\|_{2, q} \leq r^{-a}\left\|x^{a} f\right\|_{2, q} .
$$

So,

$$
\left(\int_{E}\left|F_{q}(f)(\lambda)\right|^{2} d_{q} \mathcal{\Lambda}\right)^{1 / 2} \leq\left(\frac{\tilde{c}_{q}}{\sqrt{[1-2 a]_{q}}}|E|^{1 / 2} r^{1 / 2-a}+r^{-a}\right)\left\|x^{a} f\right\|_{2, q}
$$

The desired result is obtained by minimizing the right-hand side of the previous inequality over $r>0$. 
Corollary 4.2. For $0<a<1 / 2$ and $b>0$, there is a constant $K_{a, b}$ such that for all $f \in L_{q}^{2}\left(\mathbb{R}_{q,+}\right)$, one has

$$
\|f\|_{2, q}^{(a+b)} \leq K_{a, b}\left\|x^{a} f\right\|_{2, q}^{b}\left\|\lambda^{b} \mathcal{F}_{q}(f)\right\|_{2, q}^{a} .
$$

Proof. For $r>0$, put $E_{r}=\left[0, r\left[\cap \mathbb{R}_{q,+}\right.\right.$ and $\widetilde{E}_{r}=\left[r,+\infty\left[\cap \mathbb{R}_{q,+}\right.\right.$. It is easy to see that $E_{r}$ is a bounded subset of $\mathbb{R}_{q,+}$ and $\left|E_{r}\right| \leq r$.

Then, from the Plancherel formula and Theorem 4.1, we have

$$
\begin{aligned}
\|f\|_{2, q}^{2} & =\left\|\mathscr{F}_{q}(f)\right\|_{2, q}^{2} \\
& =\int_{E_{r}}\left|\mathcal{F}_{q}(f)\right|^{2}(\lambda) d_{q} \lambda+\int_{\widetilde{E}_{r}}\left|\mathcal{F}_{q}(f)\right|^{2}(\lambda) d_{q} \lambda \\
& \leq K r^{2 a}\left\|x^{a} f\right\|_{2, q}^{2}+r^{-2 b}\left\|\lambda^{b} \mathcal{F}_{q}(f)\right\|_{2, q}^{2} .
\end{aligned}
$$

Choosing $r>0$ so as to minimize the right-hand side of the inequality, we obtain $\|f\|_{2, q}^{2} \leq$ $\left(K_{a, b}\left\|x^{a} f\right\|_{2, q}^{b}\left\|\lambda^{b} \mathcal{F}_{q}(f)\right\|_{2, q}^{a}\right)^{2 /(a+b)}$, with $K_{a, b}=\left((a / b)^{b /(a+b)}+(b / a)^{a /(a+b)}\right)^{(a+b) / 2} K^{b / 2}$, and $K$ is the constant given in Theorem 4.1.

In the same way, one can prove the following local uncertainty principle for the $q$ Fourier-sine transform.

Theorem 4.3. If $0<a<1 / 2$, there is a constant $K^{\prime}=K^{\prime}(a, q)$ such that for all bounded subset $E$ of $\mathbb{R}_{q,+}$ and all $f \in L_{q}^{2}\left(\mathbb{R}_{q,+}\right)$, one has

$$
\int_{E}\left|{ }_{q} \mathcal{F}(f)(\lambda)\right|^{2} d_{q} \lambda \leq K^{\prime}|E|^{2 a}\left\|x^{a} f\right\|_{2, q^{\prime}}^{2}
$$

where $K^{\prime}=\left(\left(\widetilde{c}_{q} / \sqrt{[1-2 a]_{q}}\right)((1-2 a) / 2 q a)\right)^{4 a}[1+2 q a /(1-2 a)]^{2}$.

Corollary 4.4. For $0<a<1 / 2$ and $b>0$, there is a constant $K_{a, b}^{\prime}$ such that for all $f \in L_{q}^{2}\left(\mathbb{R}_{q,+}\right)$, one has

$$
\|f\|_{2, q}^{(a+b)} \leq K_{a, b}^{\prime}\left\|x^{a} f\right\|_{2, q}^{b}\left\|\lambda^{b}{ }_{q} \mathcal{F}(f)\right\|_{2, q^{\prime}}^{a}
$$

with $K_{a, b}^{\prime}=\left((a / b)^{b /(a+b)}+(b / a)^{a /(a+b)}\right)^{(a+b) / 2}\left(K^{\prime}\right)^{b / 2} q^{-(a+b)}$.

Proof. The same steps of Corollary 4.2 give the result.

Theorem 4.5. If $a>1 / 2$, there is a constant $K_{1}=K_{1}(a, q)$ such that for all bounded subset $E$ of $\mathbb{R}_{q,+}$ and $f \in L_{q}^{2}\left(\mathbb{R}_{q,+}\right)$, one has

$$
\begin{aligned}
& \int_{E}\left|F_{q}(f)(\lambda)\right|^{2} d_{q} \lambda \leq K_{1}|E|\|f\|_{2, q}^{(2-1 / a)}\left\|x^{a} f\right\|_{2, q}^{1 / a}, \\
& \int_{E}\left|F_{q}(f)(\lambda)\right|^{2} d_{q} \lambda \leq K_{1}|E|\|f\|_{2, q}^{(2-1 / a)}\left\|x^{a} f\right\|_{2, q}^{1 / a} .
\end{aligned}
$$


The proof of this result needs the following lemmas.

Lemma 4.6. Suppose $a>1 / 2$, then for all $f \in L_{q}^{2}\left(\mathbb{R}_{q,+}\right)$, such that $x^{a} f \in L_{q}^{2}\left(\mathbb{R}_{q,+}\right)$,

$$
\|f\|_{1, q}^{2} \leq K_{2}\left[\|f\|_{2, q}^{2}+\left\|x^{a} f\right\|_{2, q}^{2}\right]
$$

where $K_{2}=K_{2}(a, q)=(1-q)\left(\left(q^{2 a}, q^{2 a},-q,-q^{2 a-1} ; q^{2 a}\right)_{\infty} /\left(q, q^{2 a-1},-q^{2 a},-1 ; q^{2 a}\right)_{\infty}\right)$.

Proof. From [15, Example 1], and the Hölder inequality, we have

$$
\|f\|_{1, q}^{2}=\left[\int_{0}^{+\infty}\left(1+x^{2 a}\right)^{1 / 2}|f(x)|\left(1+x^{2 a}\right)^{-1 / 2} d_{q} x\right]^{2} \leq K_{2}\left[\|f\|_{2, q}^{2}+\left\|x^{a} f\right\|_{2, q}^{2}\right],
$$

where $K_{2}=\int_{0}^{+\infty}\left(1+x^{2 a}\right)^{-1} d_{q} x=(1-q)\left(\left(q^{2 a}, q^{2 a},-q,-q^{2 a-1} ; q^{2 a}\right)_{\infty} /\left(q, q^{2 a-1},-q^{2 a},-1 ; q^{2 a}\right)_{\infty}\right)$.

Lemma 4.7. Suppose $a>1 / 2$, then for all $f \in L_{q}^{2}\left(\mathbb{R}_{q,+}\right)$, such that $x^{a} f \in L_{q}^{2}\left(\mathbb{R}_{q,+}\right)$, one has

$$
\|f\|_{1, q} \leq K_{3}\|f\|_{2, q}^{(1-1 / 2 a)}\left\|x^{a} f\right\|_{2, q}^{1 / 2 a}
$$

where $K_{3}=K_{3}(a, q)=\left[2 a K_{2}(2 a q-q)^{1 / 2 a-1}\right]^{1 / 2}$.

Proof. For $s \in \mathbb{R}_{q,+}$, define the function $f_{s}$ by $f_{s}(x)=f(s x), x \in \mathbb{R}_{q,+}$.

We have $\left\|f_{s}\right\|_{1, q}=s^{-1}\|f\|_{1, q},\left\|x^{a} f_{s}\right\|_{2, q}^{2}=s^{-2 a-1}\left\|x^{a} f\right\|_{2, q}^{2}$.

Replacement of $f$ by $f_{s}$ in Lemma 4.6 gives

$$
\|f\|_{1, q}^{2} \leq K_{2}\left[s\|f\|_{2, q}^{2}+s^{-2 a+1}\left\|x^{a} f\right\|_{2, q}^{2}\right] .
$$

Now, for all $r>0$, put $\alpha(r)=\log (r) / \log (q)-E(\log (r) / \log (q))$. We have $s=\left(r / q^{\alpha(r)}\right) \in \mathbb{R}_{q,+}$ and $r \leq s<r / q$. Then, for all $r>0$,

$$
\|f\|_{1, q}^{2} \leq K_{2}\left[\frac{r}{q}\|f\|_{2, q}^{2}+r^{-2 a+1}\left\|x^{a} f\right\|_{2, q}^{2}\right] .
$$

The right-hand side of this inequality is minimized by choosing

$$
r=(2 a-1)^{1 / 2 a} q^{1 / 2 a}\|f\|_{2, q}^{-1 / a}\left\|x^{a} f\right\|_{2, q}^{1 / a}
$$

When this is done, we obtain the result.

Proof of Theorem 4.5. Since the proofs of the two statements are similar, it is sufficient to prove (4.11).

Let $E$ be a bounded subset of $\mathbb{R}_{q,+}$. When the right-hand side of the inequality (4.11) is finite, Lemma 4.6 implies that $f \in L_{q}^{1}\left(\mathbb{R}_{q,+}\right)$; so $\mathcal{F}_{q}(f)$ is defined and bounded on $\mathbb{R}_{q,+}$. Using 
Proposition 3.1, Lemma 4.7, and the fact that

$$
\int_{E}\left|\mathcal{F}_{q}(f)(\lambda)\right|^{2} d_{q} \lambda \leq|E|\left\|\mathcal{F}_{q}(f)\right\|_{\infty, q}^{2}
$$

we obtain the result with $K_{1}=\left(\left(1+q^{-1}\right) / \Gamma_{q^{2}}^{2}(1 / 2)(q ; q)_{\infty}^{4}\right) K_{3}^{2}$.

Remark 4.8. By the same technique as in the proof of Corollary 4.2, we can show that Theorem 4.5 leads to inequalities (4.6) and (4.9) with some different constants.

\subsection{Local uncertainty principles for the q-Bessel-Fourier transform}

The $q$-Bessel-Fourier transform is defined (see [16]) for $f \in L_{q}^{1}\left(\mathbb{R}_{q,+}, x^{2 v+1} d_{q} x\right)$ by

$$
\mathcal{F}_{v, q}(f)(\lambda)=c_{v, q} \int_{0}^{\infty} f(x) j_{v}\left(\lambda x ; q^{2}\right) x^{2 v+1} d_{q} x
$$

where

$$
j_{v}\left(z ; q^{2}\right)=\left(1-q^{2}\right)^{v} \Gamma_{q^{2}}(v+1)\left((1-q) q^{-1} z\right)^{-v} J_{v}\left((1-q) q^{-1} z ; q^{2}\right)
$$

is the normalized third Jackson $q$-Bessel function, and

$$
c_{v, q}=\frac{\left(1+q^{-1}\right)^{-v}}{\Gamma_{q^{2}}(v+1)} .
$$

It was shown in [10] that for $v \geq-1 / 2$, we have the following result.

Theorem 4.9. (1) For $f \in L_{q}^{1}\left(\mathbb{R}_{q,+}, x^{2 v+1} d_{q} x\right)$, one has $\mathcal{F}_{v, q}(f) \in L_{q}^{\infty}\left(\mathbb{R}_{q,+}\right)$ and

$$
\left\|\mathcal{F}_{v, q}(f)\right\|_{\infty, q} \leq \frac{c_{v, q}}{\left(q ; q^{2}\right)_{\infty}^{2}}\|f\|_{1, v, q} .
$$

(2) $\mathcal{F}_{v, q}$ is an isomorphism of $L_{q}^{2}\left(\mathbb{R}_{q,+}, x^{2 v+1} d_{q} x\right)$ onto itself, $\mathcal{F}_{v, q}^{-1}=q^{4 v+2} \mathcal{F}_{v, q}$, and one has the following Plancherel formula:

$$
\forall f \in L_{q}^{2}\left(\mathbb{R}_{q,+}, x^{2 v+1} d_{q} x\right), \quad\left\|\mp_{v, q}\right\|_{2, v, q}=q^{2 v+1}\|f\|_{2, v, q} .
$$

The following result states a local uncertainty principle for the q-Bessel-Fourier transform.

Theorem 4.10. For $v \geq-1 / 2$ and $0<a<v+1$, there is a constant $K_{a, v}=K(a, v, q)$ such that for all $f \in L_{q}^{2}\left(\mathbb{R}_{q,+}, x^{2 v+1} d_{q} x\right)$ and all bounded subset $E$ of $\mathbb{R}_{q,+}$ one has

$$
\int_{E}\left|\mathcal{F}_{v, q}(f)(\lambda)\right|^{2} \lambda^{2 v+1} d_{q} \lambda \leq K_{a, v}|E|_{v}^{a /(v+1)}\left\|x^{a} f\right\|_{2, v, q}^{2} .
$$

Here, $|E|_{v}=\int_{0}^{\infty} X_{E}(x) x^{2 v+1} d_{q} x, \tilde{c}_{v, q}=c_{v, q} /\left(q ; q^{2}\right)_{\infty}^{2}$, and

$$
K_{a, v}=\left(\frac{\tilde{c}_{v, q}}{\sqrt{[2 v+2-2 a]_{q}}}\right)^{2 a /(v+1)}\left[\left(\frac{a q^{2 v+1}}{v+1-a}\right)^{1-a /(v+1)}+q^{2 v+1}\left(\frac{a q^{2 v+1}}{v+1-a}\right)^{-a /(v+1)}\right]^{2} .
$$


Ahmed Fitouhi et al.

Proof. Let $v \geq-1 / 2,0<a<v+1, f \in L_{q}^{2}\left(\mathbb{R}_{q,+}, x^{2 v+1} d_{q} x\right)$, and let $E$ be a bounded subset of $\mathbb{R}_{q,+}$. For $r>0$, we have, since $f \cdot x_{r} \in L_{q}^{1}\left(\mathbb{R}_{q,+}, x^{2 v+1} d_{q} x\right)$,

$$
\begin{aligned}
& \left(\int_{E}\left|\mathcal{F}_{v, q}(f)(\lambda)\right|^{2} \lambda^{2 v+1} d_{q} \lambda\right)^{1 / 2}=\left\|\mathcal{F}_{v, q}(f) X_{E}\right\|_{2, v, q} \\
& \leq\left\|\mathcal{F}_{v, q}\left(f \cdot X_{r}\right) \chi_{E}\right\|_{2, v, q}+\left\|\mathcal{F}_{v, q}\left(f \cdot \tilde{X}_{r}\right) \chi_{E}\right\|_{2, v, q} \\
& \leq|E|_{v}^{1 / 2}\left\|\mathcal{F}_{v, q}\left(f \cdot x_{r}\right)\right\|_{\infty, q}+\left\|\mathcal{F}_{v, q}\left(f \cdot \tilde{X}_{r}\right)\right\|_{2, v, q} \text {. }
\end{aligned}
$$

However, by the use of the Hölder inequality, we obtain

$$
\begin{aligned}
\left\|\mathcal{F}_{v, q}\left(f \cdot x_{r}\right)\right\|_{\infty, q} & \leq \tilde{c}_{v, q}\left\|f \cdot x_{r}\right\|_{1, q} \\
& =\tilde{c}_{q}\left\|x^{-a} X_{r} \cdot x^{a} f\right\|_{1, v, q} \\
& \leq \tilde{c}_{v, q}\left\|x^{-a} X_{r}\right\|_{2, v, q}\left\|x^{a} f\right\|_{2, v, q} .
\end{aligned}
$$

Now, if $k$ is the integer such that $q^{k} \leq r<q^{k-1}$, we get, since $a<v+1$,

$$
\left\|x^{-a} X_{r}\right\|_{2, v, q}^{2}=\int_{0}^{\infty} x^{-2 a} X_{r}(x) x^{2 v+1} d_{q} x=\int_{0}^{q^{k}} x^{2 v+1-2 a} d_{q} x=\frac{q^{2 k(v+1-a)}}{[2 v+2-2 a]_{q}} \leq \frac{r^{2(v+1-a)}}{[2 v+2-2 a]_{q}} .
$$

Then,

$$
\left\|\Psi_{v, q}\left(f \cdot x_{r}\right)\right\|_{\infty, q} \leq \frac{\tilde{c}_{v, q}}{\sqrt{[2 v+2-2 a]_{q}}} r^{(v+1-a)}\left\|x^{a} f\right\|_{2, v, q}
$$

On the other hand, since $f \in L_{q}^{2}\left(\mathbb{R}_{q,+}, x^{2 v+1} d_{q} x\right)$, we have $f \cdot \widetilde{X}_{r} \in L_{q}^{2}\left(\mathbb{R}_{q,+}, x^{2 v+1} d_{q} x\right)$, and by the Plancherel formula (4.23), we obtain

$$
\begin{aligned}
\left\|\mathcal{F}_{v, q}\left(f \cdot \tilde{x}_{r}\right)\right\|_{2, v, q} & =q^{2 v+1}\left\|f \cdot \tilde{x}_{r}\right\|_{2, v, q}=q^{2 v+1}\left\|x^{-a} \tilde{X}_{r} \cdot x^{a} f\right\|_{2, v, q} \\
& \leq q^{2 v+1}\left\|x^{-a} \tilde{X}_{r}\right\|_{\infty, q}\left\|x^{a} f\right\|_{2, q} \leq q^{2 v+1} r^{-a}\left\|x^{a} f\right\|_{2, v, q} .
\end{aligned}
$$

So,

$$
\left(\int_{E}\left|\mathcal{F}_{v, q}(f)(\lambda)\right|^{2} \lambda^{2 v+1} d_{q} \lambda\right)^{1 / 2} \leq\left(\frac{\tilde{c}_{v, q}}{\sqrt{[2 v+2-2 a]_{q}}}|E|_{v}^{1 / 2} r^{(v+1-a)}+q^{2 v+1} r^{-a}\right)\left\|x^{a} f\right\|_{2, v, q} .
$$

By minimization of the right-hand side of the previous inequality over $r>0$ and by easy computation, we obtain the desired result. 
Theorem 4.11. For $v \geq-1 / 2$ and $a>v+1$, there exists a constant $K_{a, v}^{\prime}$ such that for all bounded subset $E$ of $\mathbb{R}_{q,+}$ and all $f$ in $L_{q}^{2}\left(\mathbb{R}_{q,+}, x^{2 v+1} d_{q} x\right)$, one has

$$
\int_{E}\left|\mathcal{F}_{v, q}(f)(\lambda)\right|^{2} \lambda^{2 v+1} d_{q} \lambda \leq K_{a, v}^{\prime}|E|\|f\|_{2, v, q}^{2(1-(v+1) / a)}\left\|x^{a} f\right\|_{2, v, q}^{2((v+1) / a)} .
$$

Proof. Since $a>v+1$, the same steps as in the proof of Theorem 4.5 and the relation (4.22) give the result with

$$
\begin{aligned}
K_{a, v}^{\prime} & =\frac{\left(q^{2 a}, q^{2 a},-q^{2 v+2},-q^{2(a-v-1)} ; q^{2 a}\right)_{\infty}}{\left(q^{2 v+2}, q^{2(a-v-1)},-q^{2 a},-1 ; q^{2 a}\right)_{\infty}} c_{v, q^{\prime}}^{\prime} \\
c_{v, q}^{\prime} & =(1-q)\left(\frac{c_{v, q}}{\left(q ; q^{2}\right)_{\infty}^{2}}\right)^{2}\left(\frac{a}{v+1}-1\right)^{(v+1) / a}\left(\frac{a}{a-v-1}\right) q^{-2(v+1)((a-v-1) / a)} .
\end{aligned}
$$

Corollary 4.12. For $v \geq-1 / 2$ and $a, b>0$, there is a constant $K_{a, b, v}=K(a, b, v, q)$ such that for all $f \in L_{q}^{2}\left(\mathbb{R}_{q,+}, x^{2 v+1} d_{q} x\right)$, one has

with

$$
\|f\|_{2, v, q}^{(a+b)} \leq K_{a, b, v}\left\|x^{a} f\right\|_{2, v, q}^{b}\left\|\lambda^{b} \mathcal{F}_{v, q}(f)\right\|_{2, v, q^{\prime}}^{a}
$$

$$
K_{a, b, v}=\left\{\begin{array}{c}
{\left[\left(\frac{b}{a}\right)^{a /(a+b)}+\left(\frac{a}{b}\right)^{b /(a+b)}\right]^{(a+b) / 2}\left(K_{a, v}\right)^{b / 2} \frac{q^{-(2 v+1)(a+b)}}{\left([2 v+2]_{q}\right)^{a b / 2(v+1)}}} \\
\text { if } a<v+1, \\
\left(\frac{K_{a, v}^{\prime}}{[2 v+2]_{q}}\right)^{a b /(2 v+2)}\left(q^{-(4 v+2)}\left[\left(\frac{b}{v+1}\right)^{(v+1) /(v+b+1)}+\left(\frac{b}{v+1}\right)^{-b /(v+b+1)}\right]\right)^{a(v+b+1) / 2(v+1)} \\
\text { if } a>v+1,
\end{array}\right.
$$

where $K_{a, v}\left(r e s p ., K_{a, v}^{\prime}\right)$ is the constant given in Theorem 4.10 (resp., Theorem 4.11).

Proof. For $r>0$, we put $E_{r}=\left[0, r\left[\cap \mathbb{R}_{q,+}\right.\right.$ and $\widetilde{E}_{r}=\left[r,+\infty\left[\cap \mathbb{R}_{q,+}\right.\right.$.

We have $E_{r}$ is a bounded subset of $\mathbb{R}_{q,+}$ and $\left|E_{r}\right|_{v} \leq r^{2 v+2} /[2 v+2]_{q}$. Then, the Plancherel formula (4.23) and Theorems 4.10 and 4.11 lead to

$$
\begin{aligned}
q^{4 v+2}\|f\|_{2, v, q}^{2} & =\left\|\mathcal{F}_{v, q}(f)\right\|_{2, v, q}^{2}=\int_{E_{r}}\left|\mathcal{F}_{v, q}(f)\right|^{2}(\lambda) \lambda^{2 v+1} d_{q} \lambda+\int_{\tilde{E}_{r}}\left|\mathcal{F}_{v, q}(f)\right|^{2}(\lambda) \lambda^{2 v+1} d_{q} \lambda \\
& \leq\left\{\begin{array}{lr}
K_{a, v}\left|E_{r}\right|_{v}^{a /(v+1)}\left\|x^{a} f\right\|_{2, v, q}^{2}+r^{-2 b}\left\|\lambda^{b} \mathcal{F}_{v, q}(f)\right\|_{2, v, q}^{2} & \text { if } a<v+1, \\
K_{a, v}^{\prime}\left|E_{r}\right|\|f\|_{2, v, q}^{2(a-v-1) / a}\left\|x^{a} f\right\|_{2, v, q}^{2(v+1) / a}+r^{-2 b}\left\|\lambda^{b} \mathcal{F}_{v, q}(f)\right\|_{2, v, q}^{2} & \text { if } a>v+1,
\end{array}\right. \\
& \leq \begin{cases}\frac{K_{a, v}}{[2 v+2]_{q}^{a /(v+1)}} r^{2 a}\left\|x^{a} f\right\|_{2, v, q}^{2}+r^{-2 b}\left\|\lambda^{b} \mathcal{F}_{v, q}(f)\right\|_{2, v, q}^{2} & \text { if } a<v+1, \\
K_{a, v}^{\prime} \frac{r^{2 v+2}}{[2 v+2]_{q}}\|f\|_{2, v, q}^{2(a-v-1) / a}\left\|x^{a} f\right\|_{2, v, q}^{2(v+1) / a}+r^{-2 b}\left\|\lambda^{b} \mathcal{F}_{v, q}(f)\right\|_{2, v, q}^{2} & \text { if } a>v+1 .\end{cases}
\end{aligned}
$$

The desired result follows by minimizing the right expressions over $r>0$. 
Remark that when $a=b=1$, we obtain a Heisenberg-Weyl-type inequality for the $q$ Bessel-Fourier transform.

Corollary 4.13. For $v \geq-1 / 2, v \neq 0$, one has for all $f \in L_{q}^{2}\left(\mathbb{R}_{q,+}, x^{2 v+1} d_{q} x\right)$,

$$
\|f\|_{2, v, q}^{2} \leq K_{1,1, v}\|x f\|_{2, v, q}\left\|\lambda \mathcal{F}_{v, q}(f)\right\|_{2, v, q} \text {. }
$$

\section{Acknowledgment}

The authors would like to thank the reviewers for their helpful remarks and constructive criticism.

\section{References}

[1] G. B. Folland and A. Sitaram, "The uncertainty principle: a mathematical survey," Journal of Fourier Analysis and Applications, vol. 3, no. 3, pp. 207-238, 1997.

[2] V. Havin and B. Jöricke, The Uncertainty Principle in Harmonic Analysis, vol. 28 of Results in Mathematics and Related Areas (3), Springer, Berlin, Germany, 1994.

[3] N. Bettaibi, A. Fitouhi, and W. Binous, "Uncertainty principles for the $q$-trigonometric Fourier transforms," Mathematical Sciences Research Journal, vol. 11, no. 7, pp. 469-479, 2007.

[4] G. Gasper and M. Rahman, Basic Hypergeometric Series, vol. 96 of Encyclopedia of Mathematics and Its Applications, Cambridge University Press, Cambridge, UK, 2nd edition, 2004.

[5] V. Kac and P. Cheung, Quantum Calculus, Universitext, Springer, New York, NY, USA, 2002.

[6] F. H. Jackson, "On a q-definite integrals," Quarterly Journal of Pure and Applied Mathematics, vol. 41, pp. 193-203, 1910.

[7] M. E. H. Ismail, “On Jacksons third q-Bessel function and q-exponentials,” preprint, 2001.

[8] T. H. Koornwinder and R. F. Swarttouw, "On $q$-analogues of the Fourier and Hankel transforms," Transactions of the American Mathematical Society, vol. 333, no. 1, pp. 445-461, 1992.

[9] F. Bouzeffour, q-Analyse harmonique, Ph.D. thesis, Faculty of Sciences of Tunis, Tunis, Tunisia, 2002.

[10] A. Fitouhi, N. Bettaibi, and W. Binous, "Inversion formulas for the $q$-Riemann-Liouville and $q$-Weyl transforms using wavelets," Fractional Calculus \& Applied Analysis, vol. 10, no. 4, pp. 327-342, 2007.

[11] L. Grafakos, Classical and Modern Fourier Analysis, Pearson Education, Upper Saddle River, NJ, USA, 2004.

[12] W. G. Faris, "Inequalities and uncertainty principles," Journal of Mathematical Physics, vol. 19, no. 2, pp. 461-466, 1978.

[13] J. F. Price, "Inequalities and local uncertainty principles," Journal of Mathematical Physics, vol. 24, no. 7, pp. 1711-1714, 1983.

[14] J. F. Price, "Sharp local uncertainty inequalities," Studia Mathematica, vol. 85, no. 1, pp. 37-45, 1986.

[15] A. Fitouhi, N. Bettaibi, and K. Brahim, "The Mellin transform in quantum calculus," Constructive Approximation, vol. 23, no. 3, pp. 305-323, 2006.

[16] A. Fitouhi, M. M. Hamza, and F. Bouzeffour, "The $q-j_{\alpha}$ Bessel function," Journal of Approximation Theory, vol. 115, no. 1, pp. 144-166, 2002. 


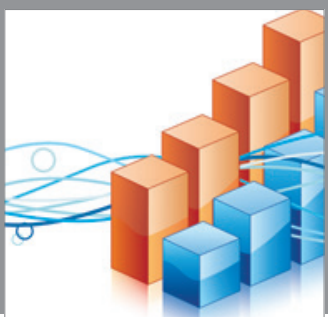

Advances in

Operations Research

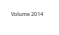

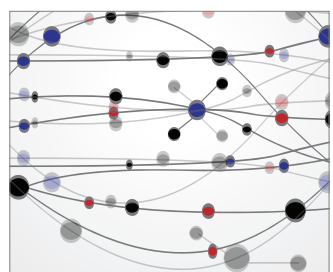

\section{The Scientific} World Journal
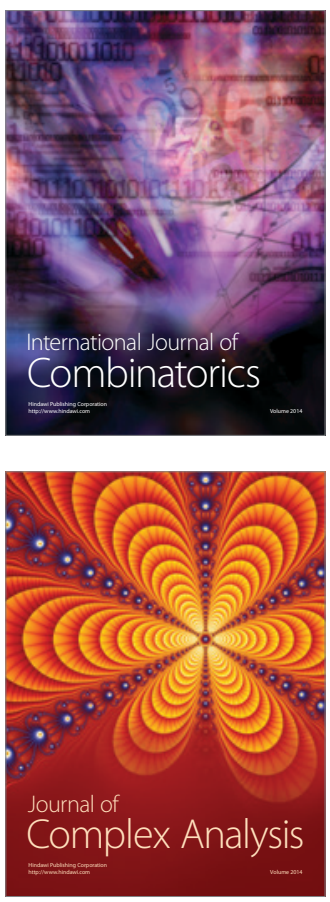

International Journal of

Mathematics and

Mathematical

Sciences
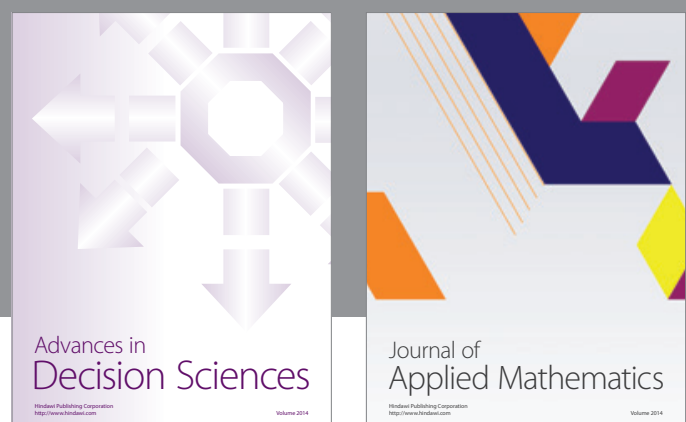

Journal of

Applied Mathematics
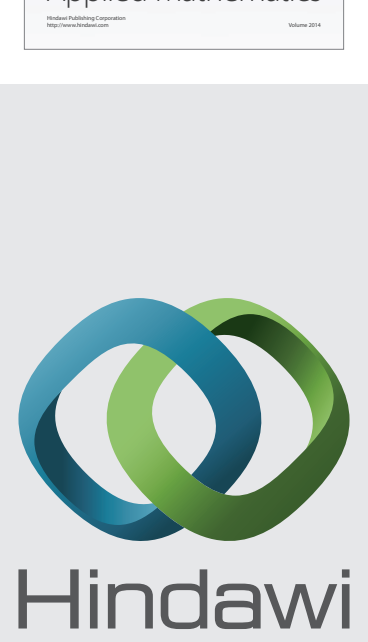

Submit your manuscripts at http://www.hindawi.com
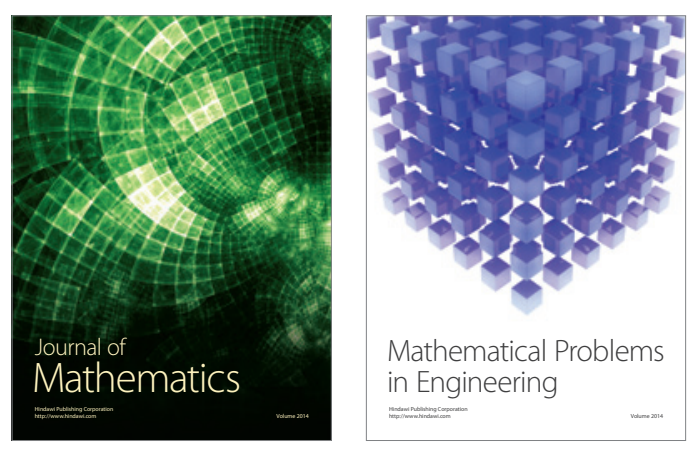

Mathematical Problems in Engineering
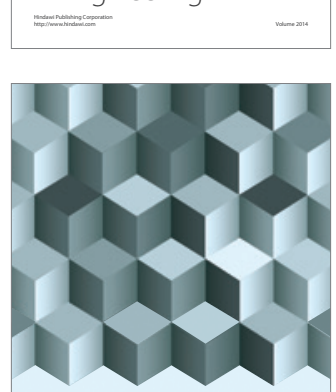

Journal of

Function Spaces
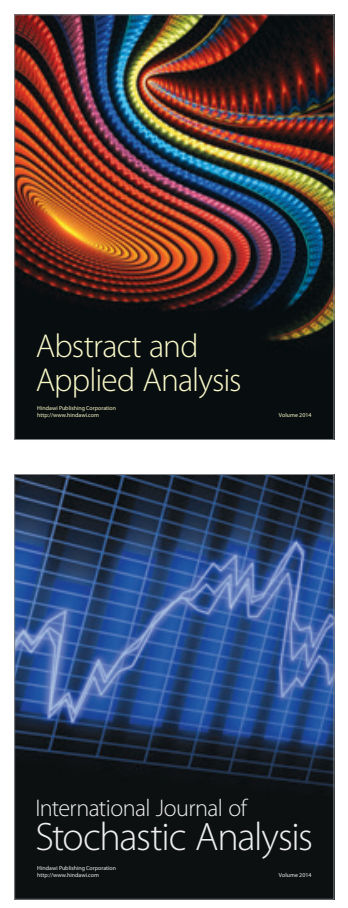

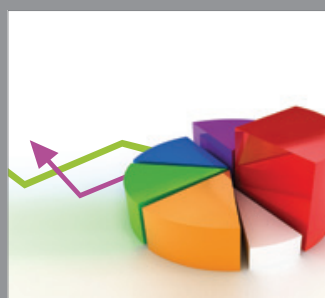

ournal of

Probability and Statistics

Promensencen
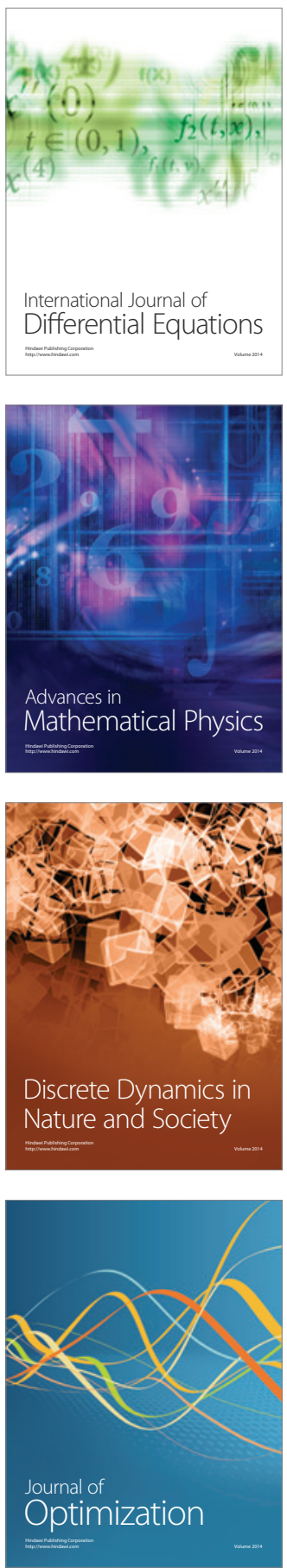